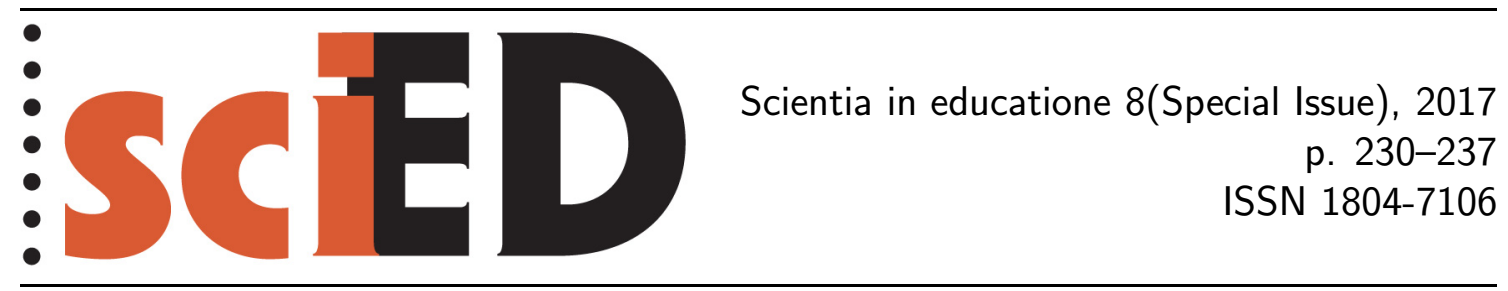

\title{
Effect of Collaborative Learning in Interactive Lecture Demonstrations (ILD) on Student Conceptual Understanding of Motion Graphs
}

\author{
Erees Queen B. Macabebe, Eleanor Alma D. Jugueta
}

\begin{abstract}
To assess effectively the influence of peer discussion in understanding concepts, and to evaluate if the conceptual understanding through Interactive Lecture Demonstrations (ILD) and collaborative learning can be translated to actual situations, ten (10) questions on human and carts in motion were presented to 151 university students comprising mostly of science majors but of different year levels. Individual and group predictions were conducted to assess the students' pre-conceptual understanding of motion graphs. During the ILD, real-time motion graphs were obtained and analysed after each demonstration and an assessment that integrates the ten situations into two scenarios was given to evaluate the conceptual understanding of the students. Collaborative learning produced a positive effect on the prediction scores of the students and the ILD with real-time measurement allowed the students to validate their prediction. However, when the given situations were incorporated to create a scenario, it posted a challenge to the students. The results of this activity identified the area where additional instruction and emphasis is necessary.
\end{abstract}




\section{INTRODUCTION}

Lecture is more often than not the most common method in teaching introductory physics. It has a relatively standard format: the teacher introduces the concept in class, solve sample problems, give practice problems to students and then give a test to assess student learning. Traditional physics instruction tends to lead students to focus more on the mathematical aspects of physics rather than on deeper conceptual understanding. It also fails to provide an active learning experience, which is essential to student learning.

One strategy that has been found effective in improving students' conceptual understanding is through interactive learning demonstrations (ILD). Various studies conducted by Thornton and Sokoloff have shown that ILDs enhance conceptual learning by motivating students to generate their own predictions and collaborate with their peers by explaining their predictions (Thornton \& Sokoloff, 1990, 1997, 1998, 2004). This engages the students to be more involved in their learning and helps them address their own misconceptions.

In most ILDs, the demonstration is set-up in front of the class with the computer display projected on a screen. The demonstration is then described to the students and they are asked to predict the outcome of the demonstration. After their prediction, the demonstration is then performed. The students immediately validate their answers whether or not they are correct by reconciling their predictions based on their observation of the demonstration.

In this study, group prediction was also employed after the individual predictions to further increase student learning of physics concepts. Before the demonstration, the students were divided into pairs or groups to discuss their individual predictions. Discussion with peers helps students learn about their own cognition given a situation. It also helps them search for alternative explanations of their predictions and modify their own thinking. Collaborative learning enhances student learning because it makes them conscious of their own thought process and helps them see how others perceive the same situation (Slavin, 1983). However, not all collaborative learning activities will result in positive learning gains. In attaining the group goal, some group discussions may be influenced by a more dominant member who does not necessarily have the correct answer. Thus, group members must be encouraged to give their maximum effort to ensure effectiveness of collaborative learning.

This study aims (1) to assess effectively the influence of peer discussion in understanding concepts presented in Interactive Lecture Demonstrations (ILD) and (2) to evaluate if the conceptual understanding through ILDs and collaborative learning can be translated to actual situations such as in human and objects in motion.

\section{Interactive LeCtURE Demonstrations}

The ILD designed for this study followed the procedure: (1) description of the demonstration, (2) prediction - individual and group predictions were conducted with each group composed of 2 or 3 students, (3) demonstration (4) discussion of results, and (5) assessment.

Human motion and cart in motion were the two set-ups considered in the ILD. Three situations were presented: (1) a person walking away from or toward the origin, (2) a cart given an initial gentle push or strong push, and (3) a mass attached 

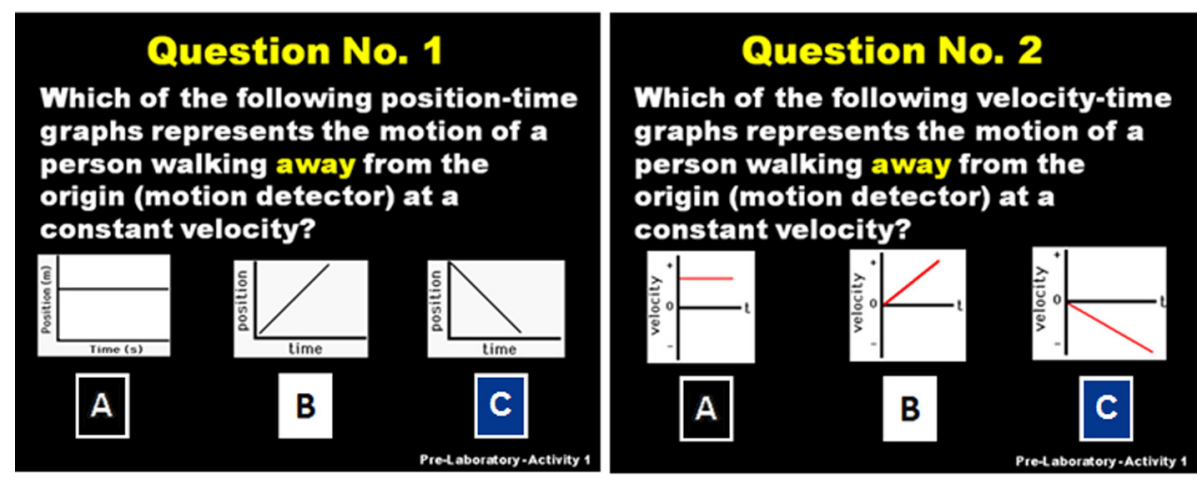

Figure 1: The slides for human motion showing how the questions were presented to the students during the individual and the group prediction

Figure 2: Set-up for cart in motion

showing the mass attached to the cart

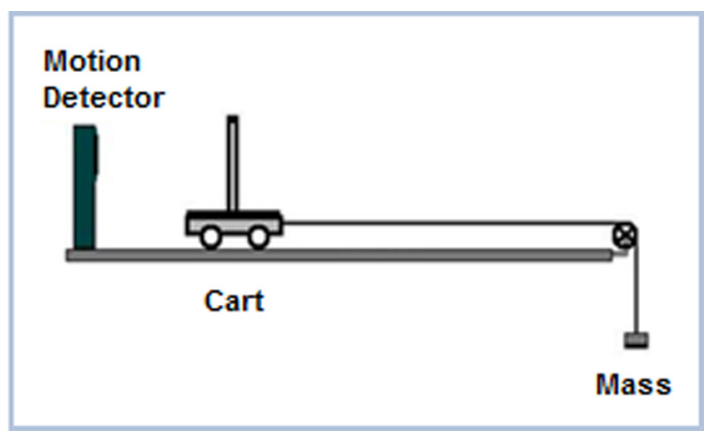

to the cart and then the cart is released from rest. Ten (10) questions on the motion graphs of these situations were asked during the individual and group prediction. Figure 1 contains two slides with questions pertaining to human motion graphs. It shows how the questions and the situations were presented to the students. Figure 2 is the diagram shown to the students to illustrate the third situation.

In the individual prediction, the students were asked to choose from a given set of graphs the one which represents the motion being described. They were then grouped and the same slides with the question and the choices were shown. This time the students were allowed to discuss their individual prediction and based on their discussion, they were required to come up with a common answer.

To understand the different motion graphs and, to analyze and interpret the motion graphs, real-time data acquisition tools were utilized in the ILD. A motion sensor interfaced to a computer with LoggerPro ${ }^{\mathrm{TM}}$ via LabPro ${ }^{\mathrm{TM}}$ was used to obtain the motion graphs. During the lecture demonstration, the position vs. time $(p-t)$ and the velocity vs. time $(v-t)$ graphs of each situation were plotted. The realtime graphs provide the correct answer to the prediction question. A discussion of the graphs and analysis of the motion in relation to the graphs followed after each demonstration.

To evaluate the conceptual understanding of the students, an assessment that integrates the ten situations into two scenarios was given. This was conducted immediately after the ILD so no reinforcement or in-class discussions were conducted prior to assessment. In the assessment, they were asked to draw the $p$ - $t$ and the $v$ - $t$ graphs.

The first scenario was described as follows: A person (1) walks from the detector slowly and steadily for $6 \mathrm{sec}$, (2) then stands still for $6 \mathrm{sec},(3)$ and then walks toward the detector steadily about twice as fast as before. The set-up for the second 
Figure 3: Illustration and caption of the second scenario in the final assessment

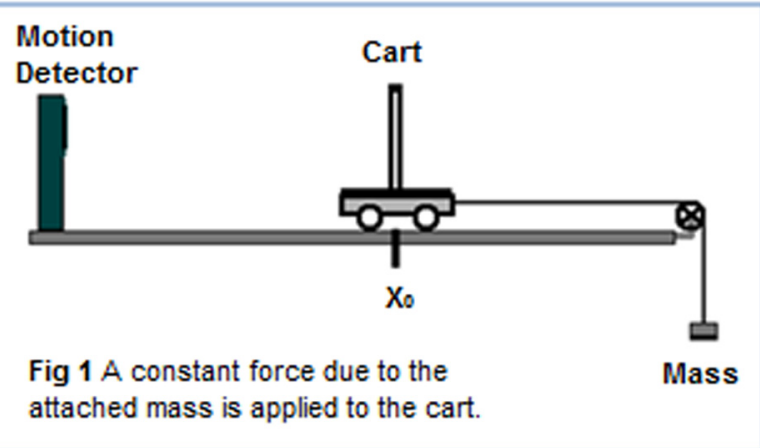

scenario is shown in Figure 3 where a string with a hanging mass at one end was attached to a cart giving it a constant force. The scenario was described as: the cart was given an initial push towards the left. (1) At $t_{0}$, the cart is at $x_{0}$ and moves toward the motion detector from $t_{0}$ to $t_{1}$. (2) Then, the cart moves away from the motion detector from $t_{1}$ and is back at $x_{0}$ at $t_{2}$, (3) Passing through $x_{0}$, continues to move away from the motion detector until $t_{3}$.

The ILD and the corresponding assessment were administered to 151 university students comprising mostly of science majors but of different year levels. This was conducted within the first week at the beginning of their first Physics course in the university. Thus, we assume that the students did not receive introductory lecture on motion graphs prior to the ILD.

\section{INDIVIDUAL AND GROUP PREDICTIONS}

Analysis of the results shows a significant increase in the number of correct answers after peer discussion. Figure 4 shows the graphs of (a) the percentage of students and their answer in each item in the individual prediction and (b) in the group prediction. In human motion, questions 1 and 2 are shown in Figure 1, and questions 3 and 4

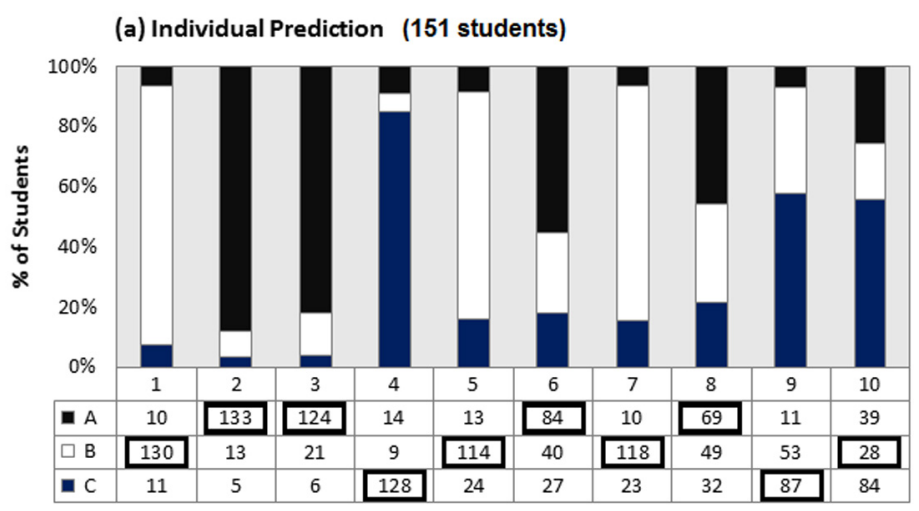

Figure 4: Plots of the percentage of students who answered either A, B, or C, in each question ( $x$-axis), (a) in the individual prediction and (b) in the group prediction. The boxed numbers indicate the correct answers

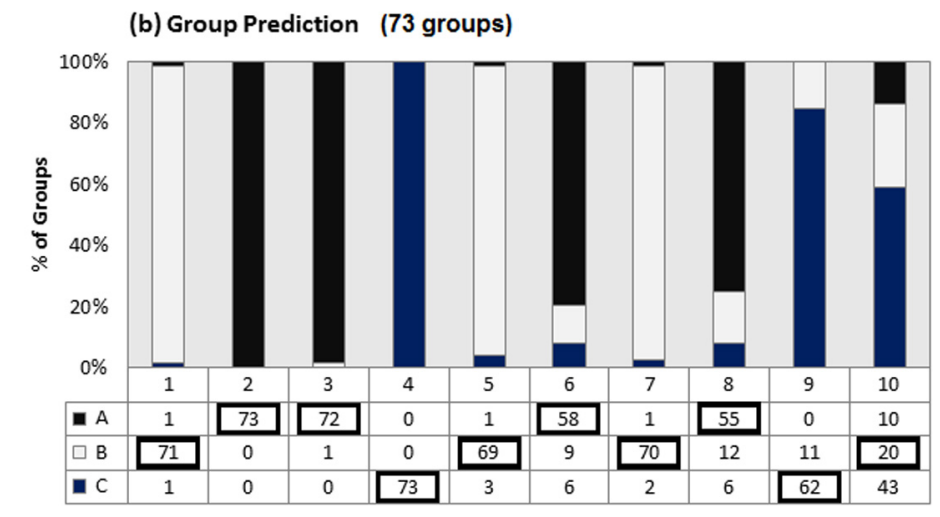


asked the students to choose the $p$ - $t$, and the $v$ - $t$ graphs, respectively, of a person walking towards the motion detector. From an average of $85.26 \%$ in the individual prediction, the average number of correct answers increased to $99.01 \%$ after peer discussion, an improvement of $16.12 \%$.

From the individual prediction, many found difficulty in visualizing the $v$ - $t$ graph of an object given an initial push (questions 6 and 8) moving along a frictionless track as described by the second situation. It is possible that the students failed to connect the meaning of "initial push" in this situation. Thus, their answers to the questions were derived from a possible misconception which implies that an external force is always present in this scenario. The said external force can be due to the initial force which, by Newton's Laws of Motion, causes the object to accelerate thereby increasing the velocity of the cart. After the group discussion, the number of students with correct answers in these questions increased by $23.18 \%$ and $29.80 \%$, respectively.

Questions 9 and 10 pertain to the third situation shown in Figure 2. Of the 87 who answered (C) nonlinear increase in question 9 , the $p$ - $t$ plot of the cart, only 17 answered (B) linear increase in question 10 which asked for the $v$ - $t$ plot of the motion. After the group discussion, there is a significant improvement in the number of correct answers in question $9,85.43 \%$ from $57.62 \%$. However, the increase in question 10 is only $9.27 \%$, from $18.54 \%$ to $27.81 \%$. Also, of the 17 who got the correct answer in question 10 in the individual prediction, 5 changed their answers in the group prediction. It means that these students were not confident with their answer and was easily convinced by their peer in the group prediction. Overall, however, the improvements observed in the total score of the groups and the item scores seen in Figure 4 indicate the positive effect of collaborative learning.

\section{Assessment}

The achievement gain between prediction and assessment were obtained and analyzed. In the assessment, some items were similar to the situations given in the prediction and ILD. They were the basis for the achievement gain analysis.

Figure 5 shows the assessment sheet with the correct answers. In Figure 6, the graph of the percentage of students with correct and incorrect answers in the final assessment is presented. The segments of the motion graphs in Figure 5 were

ACTIVITY 1: Human Motion
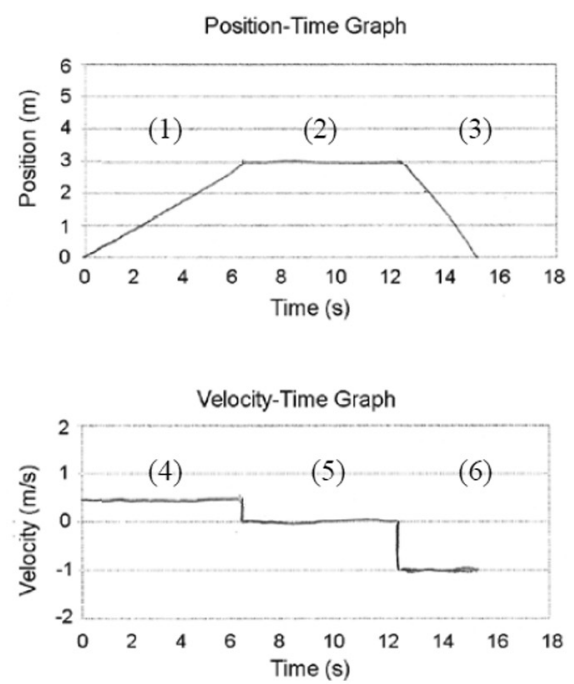

ACTIVITY 2: Cart in Motion
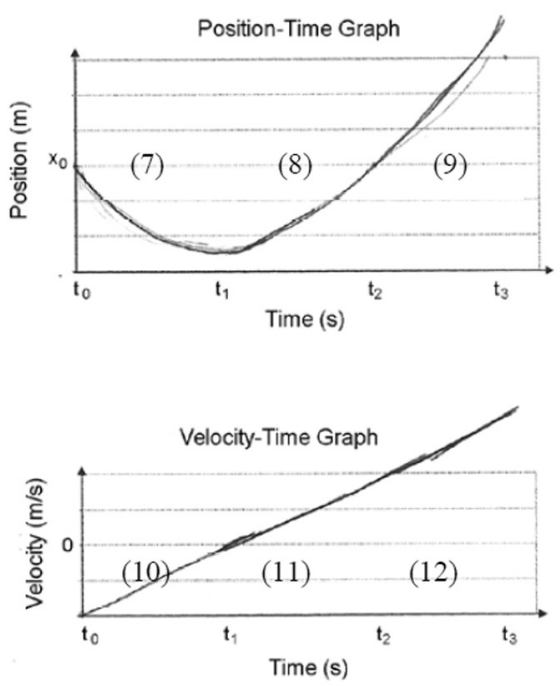

Figure 5: The graphs drawn by one of the students in the assessment. Each segment of the plots was given a corresponding item number 


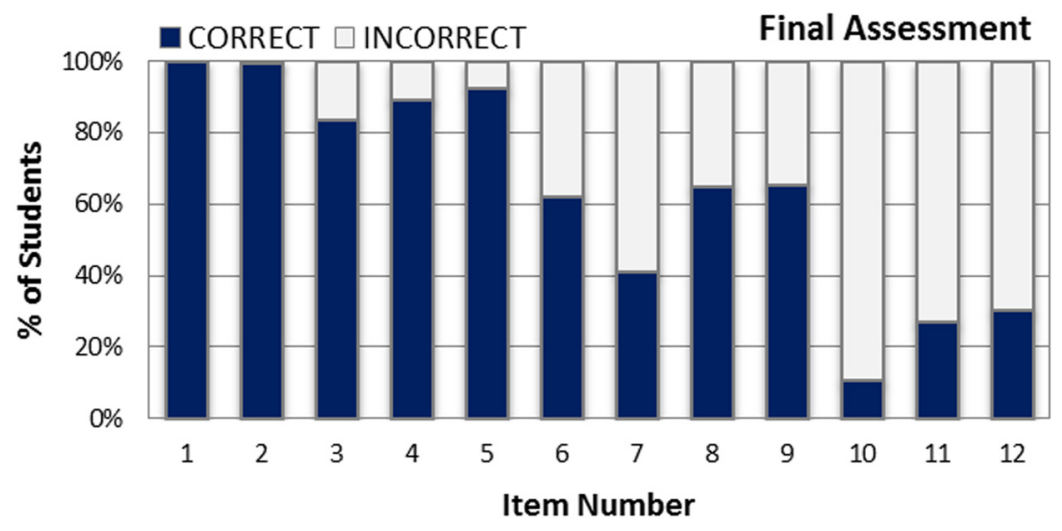

Figure 6: The graph of the percentage of students with correct and incorrect answers for each item in the final assessment

Table 1: Mapping of the items in the assessment that correspond to or is similar to the questions in the prediction part of the ILD, the percentage of students with correct answers and the achievement gain for each corresponding items

\begin{tabular}{|c|c|c|c|c|c|c|c|c|c|c|}
\hline Item & 1 & \multicolumn{2}{|c|}{3} & 4 & \multicolumn{2}{|c|}{6} & 8 & 9 & 11 & 12 \\
\hline Assessment (\%) & 100 & \multicolumn{2}{|c|}{83.44} & 89.40 & \multicolumn{2}{|c|}{62.25} & 64.90 & 65.56 & 27.15 & 30.46 \\
\hline Question & 1 & 3 & 6 & 2 & 4 & 7 & & 9 & & 0 \\
\hline Ind. Prediction (\%) & 86.09 & $\overline{82.12}$ & 55.63 & 88.08 & 84.77 & 78.15 & 57 & 61 & & .54 \\
\hline *Gain (\%) & 13.91 & 1.32 & 27.81 & 1.32 & -22.52 & -15.90 & 7.28 & 7.95 & 8.61 & 11.92 \\
\hline
\end{tabular}

assigned a number which corresponds to the $x$-axis of the graph in Figure 6 . Table 1 shows the items in the assessment that correspond to or are similar to the questions in the prediction part of the ILD. Also, the percentage of the number of students with correct answers in the assessment and in the individual prediction are shown in the table, as well as the achievement gain for each corresponding items.

Assessment item 3 (see Figure 5) corresponds to prediction questions 3, which pertains to the trend of the $p$-t plot, and 6 , which pertains the magnitude of the plot when the velocity is doubled. The same goes with items 6 , questions 4 and 7 but they refer to $v$ - $t$ plots. In assessment item $3,10.60 \%$ of the students' answer have the correct trend (linear, $+y$-axis, - slope) but incorrect magnitude of the slope. If we take this into account, then the achievement gain between item 3 and question 3 is actually $11.92 \%$.

In item 6 , the achievement gains from questions 4 and 7 are both negative. Although $100 \%$ and $96.03 \%$ of the students were correct in questions 4 and 7 , respectively, in the group prediction, the achievement gains are negative. The assessment shows that the students know that for constant velocity, $v$ - $t$ plot is a straight horizontal line. However, $18.54 \%$ did not take into account the direction of the motion even though this was emphasized in the discussion that followed the demonstration.

In the second scenario, items 7 and 10 were introduced to evaluate if the students can already integrate the motion towards the origin while a constant force in the opposite direction is in effect. Although the percentages of correct answers in this scenario are low as seen in Figure 6, the achievement gain is positive. About $25.83-33.78 \%$ of the students considered the $p$ - $t$ plot to be linear, which was the common mistake in these items. In the $v$ - $t$ plot, $39.07 \%$ of the students represented 


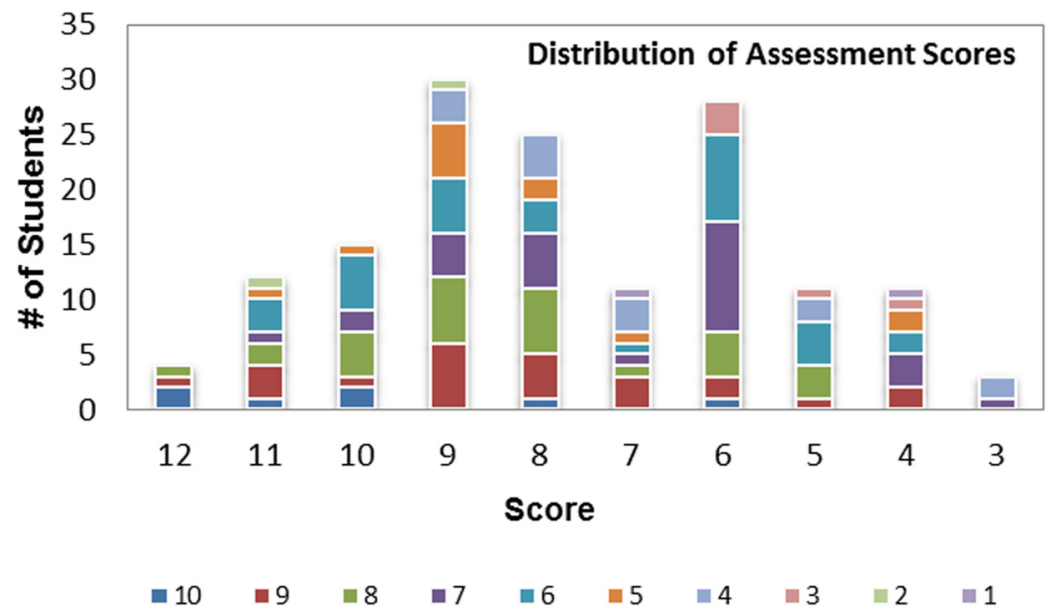

Figure 7: Plot of the raw scores that the students obtained in the assessment. The legend at the bottom of the graph indicates the raw score of the student in the individual prediction

item 10 as a linear plot with negative slope located below the $x$-axis. As a result, their plots in items 11 and 12 were automatically shifted although it is evident in their answers that they remember the trend of the $v$ - $t$ graph of the cart being pulled by the hanging mass.

Figure 7 shows the plot of the raw score the students obtained in the assessment. It also shows the raw score these students obtained in the individual prediction (indicated in the legend). The minimum score in the assessment should be four (4) since items 1, 3, 4, and 6 were in the ILD. Unfortunately, this is not the case as seen in Figure 7. Also, some students achieved negative gain between the individual prediction and the assessment scores. One possibility is that their predictions were just guesses since the choices were given and, when asked to draw the graph, they failed to interpret the motion because they did not grasp the concepts during the group discussion and even after ILD. Looking at the figure, $72.41 \%$ who got a score of 6 were correct it items 1-6 only, while $72.73 \%$ of those who got 7 were correct in items 8 and 9 . However, their plots in items 11 and 12 were shifted down but followed the correct trend. This is also true for the $v$ - $t$ plots of $65.45 \%$ of those who got a score of 8 or 9 . This could indicate that those students with scores falling between 7 and 9 learned from the group discussion and the ILD but did not know how to plot the motion of item 10. In general, the assessment results show improved scores for most of the students.

\section{CONCLUSiON}

In an Interactive Lecture Demonstration, collaborative learning produced a positive effect on the prediction scores of the students. The ILD with real-time measurement allowed the students to validate their prediction. However, when the given situations were incorporated to create a scenario, it posted a challenge to the students. The results of this activity identified the area where additional instruction and emphasis is necessary. In particular, Newton's second law of motion, in relation to the situation where the acceleration due to the applied force and the velocity of the body are in the opposite direction, needs to be elaborated. 


\section{REFERENCES}

Slavin, R. E. (1983). When does cooperative learning increase student achievement? Psychological Bulletin, 94, 429-445.

Thornton, R. K. \& Sokoloff, D. R. (1990). Learning motion concepts using real-time microcomputer-based laboratory tools. American Journal of Physics, 58 (9), 858-867.

Thornton, R. K. \& Sokoloff, D. R. (1997). Using interactive lecture demonstrations to create an active learning environment. The Physics Teacher, 35(6), 340-347.

Thornton, R. K. \& Sokoloff, D. R. (1998). Assessing student learning of Newton's laws: The force and motion conceptual evaluation and the evaluation of active learning laboratory and lecture curricula. American Journal of Physics, 66(4), 336-352.

Thornton, R. K. \& Sokoloff, D. R. (2004). Interactive lecture demonstrations: Active learning in introductory physics. Hoboken, NJ: Wiley.

Erees Queen B. Macabebe

Department of Electronics, Computer and Communications Engineering, Ateneo de Manila University, Loyola Heights, Quezon City 1108 Philippines

Eleanor Alma D. Jugueta

Computer Science Department, Faculty of Engineering, University of Santo Tomas, España Blvd., Manila 1008 Philippines

Department of Physics, Ateneo de Manila University, Loyola Heights, Quezon City 1108 Philippines 